\title{
Under the background of informatization of electronic archives management and innovation
}

\author{
ZHANG Jixian \\ Weifang Vocational College, Weifang, 261041, China.
}

Keywords: file management, informationization, electronic archives, management innovation

\begin{abstract}
In the era of information and network high-speed explosion, file management work to maintain synchrony with the social and economic development, deal with new problems in new ways, and make records management work better adapt to economic and social development of the information age, the article analyses how to carry out archives management improvement and mechanism innovation, actively explore the characteristics of the new new archives management work in the information age, discusses how to form the service mode of enterprise archives management innovation. Under the background of informatization, the most important is to strengthen the application and management of electronic archives, increase the intensity of research and practice, summed up a set of scientific methods of modern archives management, technology, standard, to ensure that it can serve the enterprise and society at the same time, encourage healthy and rapid economic development.
\end{abstract}

\section{Introduction}

A large number of enterprises in the process of informatization construction entities file needed less and less, replaced by electronic documents, however, the traditional archives entities face electronic file archives management mode is still on the conservative management pattern, many electronic get proper treatment cause huge loss of archives information resources, some of the archives in the face of informationization is appropriate, can take online to receive electronic files, and then to digitize entity files. But due to the unclear understanding of the fundamental aim of archives information construction, lack of scientific guidance, lead to low efficiency caused by the lack of information sharing. Field of archives management in our country, therefore, the most urgent task is to improve the innovation ability, advocating scientific work mechanism, thus for the culture of rapid economic development of the whole society to provide quality information services.

\section{The file and archive management related concepts}

The main content of the file management is to use scientific methods to collect, sort out, retained, identification file, even in late to provide the corresponding statistical data, the cause of the state and society in all kinds of services. As constantly advance the informatization construction in our country, every industry vigorously popularize the use of computer and network, makes the file management and increases the information processing and output two link, and the two link is the key of the new era of file management. The characteristics of the archives management work can be roughly divided into the following kinds. The first, involves the field widely, with a huge work. Second, contains a huge amount of information, for each domain has reference and guiding significance to the development plan. Third, because of its huge job content, in the information era ushered in the development bottleneck, from work idea to technology innovation needs further optimization. Fourth, the traditional archives management adopts linear management, can form a plane network, resulting in the loss of information, and the rapid development of information age asks to vertical, Shared file management, once the relies on the rigorous science and technology and innovation in the field of management meet the requirements, can lead to common leap type development of the industry and other industry. 


\section{Files management theory innovation}

Under the situation of thinking innovation, archives workers to carry out archives management mechanism innovation, and actively explore and master the development of archives work under informatization, gradually establish and form is helpful to archives comprehensive development and utilization of management mechanism, improve the work efficiency and management level, should first try to keep up with the part of the informatization construction, establish archives information resource collection network. With the improvement of the development of society and informatization degree, archives information is more and more big, face more and more widely, only by department of archives work cannot be fully and accurately the information collection, this requests us to fully arouse and exert the social various industries, to jointly manage large data path.

At the same time, according to the nature of the work of archives work, every task to the elaboration and quantify, formulate scientific work flow, establish and perfect the work permissions in different processes, to make every department and every practitioner of responsibility clear, have clear goals. Conducted on the basis of the clear process innovation, and actively explore and analyze the new features of archives management mode of archives work in the specified annual guidelines and goals, and employees in the work of the specific objectives, to establish a competition mechanism, to ensure that the excellent talents to stand out, at the same time give them appropriate new technical training policy, improve the level of the industry. Archives work, moreover, is a professional work, should establish innovation encouragement mechanism in the industry and encourages practitioners to advanced work methods, and create respect knowledge, respect talent working atmosphere, arouse overall work enthusiasm and creativity of archivists.

The rapid development of information technology, brought convenient for archives work, make this area for development opportunities, also make the work is facing many new challenges. Only fully seize the opportunity, vigorously by means of information science, optimization and upgrading of traditional archives management technology, to achieve the collection of archives, the respect such as using synchronous development. To the innovation of the archives carrier used in the first place, in use for many years, on the basis of paper files, bold to promote information technology using electronic archives. Because of the characteristics of the new electronic files for its quick, accurate, in document filing, sorting, identification, statistics and so on each link efficiency is prominent, the archives management workers should strengthen the study, absorb foreign experience, to form a complete set of, suitable for China's national conditions of the technology of electronic archives management.

\section{How to realize the enterprise file management innovation}

Information era, become the key of the enterprise information management, information construction is more important for enterprise file management, archival institutions not only faced with the change of thinking mode, at the same time to be able to make a change in the way management in time, file management system should be established digital archives management system, has the following specific requirements.

1)full archives information resources, construction integration of archives management platform as soon as possible.

Scientific integrated enterprise internal departments, all kinds of other archives resources is the precondition of archives management innovation work, only adjust archives resources belong to the direction, correct positioning mechanism of the nature of the work of archives work, further straighten out the work of archives management and other professional department relationship, establish a file management as the main body, the business enterprise inside other professional departments cooperate archives resource integration system, to achieve enterprise of all kinds of professional archives, no dead center management. [22] while increasing archives public service ability, to the enterprise and its subordinate units to provide "one-stop" archive retrieval and use of the service, this will not only help improve the image of the archives management departments within the enterprise, also can improve the information transformation value and public service 
level.

2)further process of digital archives construction, set up new file service platform.

As part of the enterprise, the enterprise archives management of the business enterprise inside all the public affairs related to information, and timely use of advanced technology to build digital archive is advantageous to the enterprise overall development platform, through constructing the relevant enterprise archives website, not only can provide the file information to employees, but also more widely spread the enterprise culture, increase the interaction between employees and enterprises.

3)actively expand archives information using work, enhance the level of archives in the public service.

At the same time establish corresponding rules and regulations within the enterprise, strengthening the construction of archives management, standardization and rationalization of the archives management system, to implement file management standard process. Dare to try using modern means of science and technology, the improvement work and the management way, improve information management level of enterprise archives management and overall business level.

4)At the same time of speed up the construction of electronic archives and the strengthening of electronic archives information security management, presenting work loopholes and defects of archives information compromised, leak phenomenon, security file management departments within the enterprise information construction development in a healthy environment.

Improve the staff's moral standards, combined with the corresponding rules and technical support, and ensure the safety of the archives information, so as to realize the real file information, confidentiality, to strengthen the security, achieve all-round development.

\section{File management process innovation}

Due to a huge number of archives enterprise archives use paper archives and the archives digital parallel working system in two ways, the archives work in the new process can be adapted to the modern enterprise development. In the early part of the management, file management process includes archives formation of the early management of electronic documents, data and hand over hand over archives management, the management, dynamic management of equipment files and the use of archives. After a period of time after verification, the practice of archives department to optimize workflow, in the following aspects:

(1) all of the relevant project management technical data, drawings after finishing the first time into the computer management system, to ensure the integrity of science and technology information, convenient for later stages to review and other engineering construction the reasonable use of information.

(2) in early began to file, file management and control planning, front-end control methods, this method not only directly applied to engineering in the early period of the archives management, throughout the entire working process at the same time. Perfect before because of system reason, from personnel of course of business caused the front-end control can not be used correctly, to complete the preparation, checking and transferring process of engineering archives. After working process optimization, archives can jointly with other departments directly involved in the project schedule, review all technical information collected at the same time, more standardized and timely information management.

(3) strengthen the personnel business level, improving the utilization ratio of archives, and make the business enterprise inside save a huge amount of information can directly provide service for engineering and technical personnel. Due to decreased paper files management informationization process at the same time, can reduce the archives management costs, reduce the cost.

(4) the new workflow can quicken the pace of the archives of acceptance, the maximum range for the enterprise to provide the support of the work.

The greatest characteristic of innovation of the working process of the enterprise is to realize the dynamic management of equipment files, to ensure that the file update, at the same time realize the 
version switch application system in a timely manner. Responsibility at the same time, to strengthen the archives job functions, ensuring information over the finishing clear, transparent to speed up the information utilization. Through the work process optimization, in order to strengthen the management of the department.

The presence of a large number of accounting files, for enterprise file management department of taiyuan iron and steel mainly adopt the mode of centralized and unified management, strengthen the digitization process, improving the utilization ratio of archives and sharing.

Production management archives is the confidential information of the enterprise, is also a blank area of many enterprise archives management, because some of the archives information for many years basically not really into the management system, for the production and business operation is the focus of the work process to strengthen the archives management of the centralized and unified management, in accordance with the corresponding system incorporated into the corresponding scope of archive management.

Enterprise archives management mainly through the interaction within the area, not only need each regional archives to cooperate, and to the scene related technical personnel to cooperate at the same time, to all sorts of need archives service personnel to provide services.

And warehouse file is always the omissions point of archives work, taigang in this way is to take a step to control inventory and warehouse management two aspects.

\section{Build enterprise archives service website}

Enterprise archives system to provide the online service first to set up on the basis of the database service website, provide the corresponding online query system, not only opens up a new way for utilization of information resources, and optimize the traditional entity to provide files for content service, guide the user through the network information resources. Taigang specially set up the enterprise file management department official document archive management web site, as long as the enterprise staff's work number and password login, then need real-time access, browse, the site since 2000, the business enterprise inside all of document information. Archives department under the state council in conjunction with the network department specifically for enterprise document since 2000 in the background to the corresponding categories and user permissions Settings, in improving working quality and service efficiency and guarantee the safety of document information. Enterprise document archive management website open not only work for enterprise staff provides a convenient, in the very great degree also promoted the socialization of information and resources sharing.

(1) the entirestation type information release system, support the diversified information classification. Website information set the number of categories in the building restrictions, when the name of each category can be modified in the background, thus fully meet the needs of the diversity of information and information release. Support both HTML editor, the title and content of the information displayed can be editing, on the front page sorting into artificial positioning.

(2) the optimization of information retrieval system. Site in accordance with the standards of electronic archives management requirements to establish the corresponding format and unified repository, will have open directory information and public documents in online query range. Information resources include the original catalogue database, database, etc., its vast amount of data can be either for ordinary workers, can also be in the service of professional and technical personnel, ensure that meet the demand of users widely. For search engine optimization can be embodied in two aspects, one is able to make query keywords, keyword fields including, head of the department, time, etc. Can also according to the file number, document number, project, time, etc. The second is to fuzzy query, the user can fuzzy fields, screening of the query. Site is within a certain time period to search for files at the same time, affiliation and refer to the theme of The Times and other automatic sorting.

(3) the optimization search engine system. The user can according to need, find searches, and archives department under the state council in conjunction with the department of network to carry on the backstage rights management, permissions constraints. Also can divide the maintenance 
work according to the function of website, let archives management institutions at all levels to work, file management staff shall not go, shall audit information, within the permission to upload information.

(4) file document management web site layout and columns. Layout of module layout, including five again according to the position of subdivided into chunks. Main home page placed in taiyuan iron and steel group co., LTD., set the navigation bar. Middle position conspicuous, can be placed each department information module, include pictures, text messages, multimedia information, etc. Placed below location main design website copyright, address, contact information. Position is used to set the member login plate on the left. At the same time set up links, arrange the news and archives regulations, studying achievements, such as communication platform, convenient and abundant information query to use.

\section{Conclusion}

This paper discusses the archives management and innovation model is focused on archives management, technical innovation, service three aspects, the application of archives shall be according to their own characteristics to optimize and refine. Of archives, books, information integration is the inevitable trend of archives work in the future, archives, library, information of the three subjects in the field of scientific research have a common historical origin, in the process of modernization construction of information in order to improve the efficiency, reduce the cost of scientific research and industries to promote common prosperity and development, should be in deeper development of archives, books and information integration of scientific management system, use and develop the archives information resources to the greatest extent.

\section{References}

[1] Lv Yuehua, raise the level of archives management scientific train of thought, the shenzhou 2013 (12).

[2] Liu Ye, on the analysis of archives management more scientific, business information, 2013 (41).

[3] Qiu jie, strengthen the archives management modernization, and promote modernization of highway maintenance management, 2013 (6).

[4] Dai Jingjun, improve the level of scientific management methods archives, 2013.

[5] Guo wei, improve the level of scientific archives management of route choice analysis, management, 2013 (19).

[6] Cui Wenbin, improve the level of scientific archives management of routing, 2013. 